\title{
Preclosure technique versus arterial cutdown after percutaneous cannulation for venoarterial extracorporeal membrane oxygenation
}

\author{
Abhimanyu Chandel, MD, ${ }^{a}$ Mehul Desai, MD, ${ }^{b}$ Liam P. Ryan, MD, ${ }^{c}$ Lindsay Clevenger, NP, \\ Alan M. Speir, MD, ${ }^{c}$ and Ramesh Singh, MD $^{c}$
}

ABSTRACT

Background: Arteriotomy repair through the preclosure technique during elective arterial access procedures is well documented. Outcomes associated with application of this technique to the removal of arterial access cannulas in patients undergoing urgent venoarterial extracorporeal membrane oxygenation (VA-ECMO) have not previously been reported.

Methods: We reviewed the records of consecutive patients who required VAECMO for cardiogenic shock. Patients were compared by use of the preclosure device (Perclose ProGlide Suture-Mediated Closure System; Abbott Vascular, Abbott Park, III) at time of VA-ECMO cannulation. The rate of limb complications (composite of limb ischemia, infection, and site necrosis) and secondary end points of bleeding events, pseudoaneurysm, distal part embolization, and intensive care unit length of stay after decannulation were compared between the groups.

Results: Ninety-nine consecutive patients managed with VA-ECMO were identified and the preclosure device was utilized in 51 of these patients. Preclosure device failure occurred in 5 instances (9.8\%) and was successfully managed with surgical repair in 4 cases and endovascular intervention in another. Use of the preclosure device was associated with both fewer limb complications (odds ratio, 0.19; 95\% confidence interval, 0.03-0.78) and bleeding events (odds ratio, 0.21; 95\% confidence interval, 0.04-0.89). Pseudoaneurysm $(\mathrm{n}=0)$ and distal part embolization $(n=1)$ were infrequently encountered in the cohort and no difference in intensive care unit length of stay after decannulation was noted between the groups.

Conclusions: In this cohort, use of the preclosure technique in weaning from VAECMO was technically feasible, safe, and associated with an approximate $80 \%$ lower likelihood of limb complications and bleeding events compared with surgical removal. (JTCVS Techniques 2021;10:322-30)

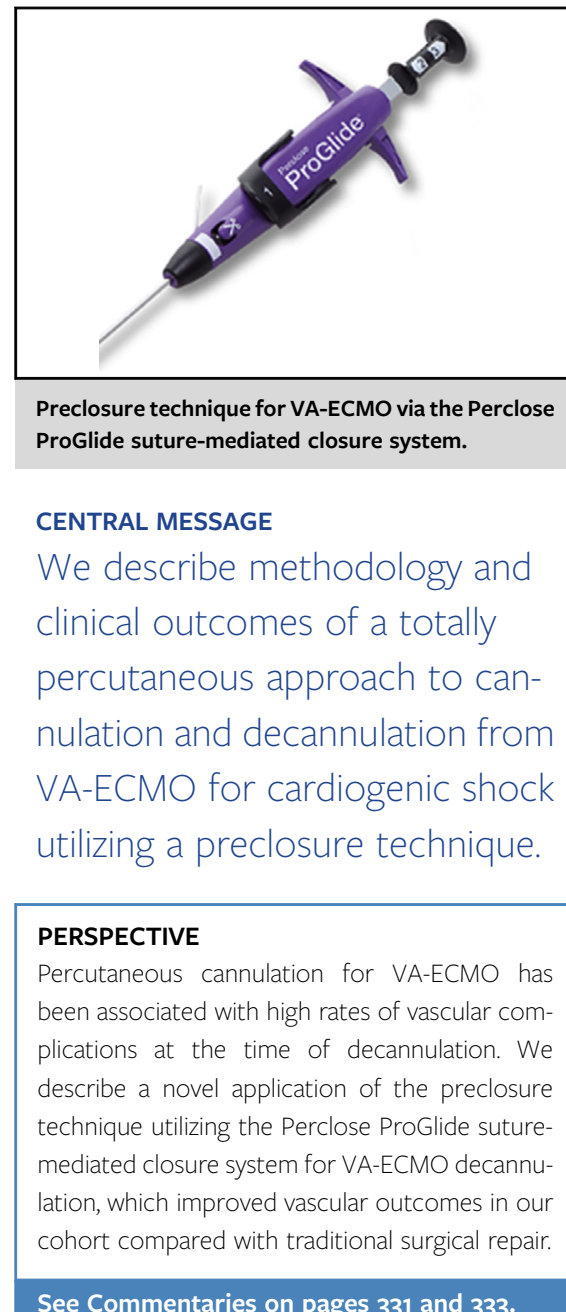

See Commentaries on pages 331 and 333 .

\footnotetext{
From the ${ }^{a}$ Department of Pulmonary and Critical Care, Walter Reed National Military Medical Center, Bethesda, Md; ${ }^{\mathrm{b}}$ Department of Critical Care, Pulmonary, Allergy, and Immunology, Inova Health System, Fairfax, Va; and ${ }^{\mathrm{c}}$ Department of Cardiac Surgery, Inova Heart and Vascular Institute, Fairfax, Va.

Received for publication March 11, 2021; accepted for publication Aug 17, 2021; available ahead of print Aug 26, 2021.

Address for reprints: Abhimanyu Chandel, MD, Department of Pulmonary and Critical Care, Walter Reed National Military Medical Center, 8901 Rockville Pike, Bethesda, MD 20814 (E-mail: Abhimanyu.chandel.mil @mail.mil).

2666-2507

Published by Elsevier Inc. on behalf of The American Association for Thoracic Surgery. This is an open access article under the CC BY-NC-ND license (http:// creativecommons.org/licenses/by-nc-nd/4.0/).

https://doi.org/10.1016/j.xjtc.2021.08.030
} 


\section{Abbreviations and Acronyms \\ ICU = intensive care unit \\ VA-ECMO $=$ venoarterial extracorporeal membrane oxygenation}

provide extended circulatory support as a bridge to patient recovery or a more durable mechanical solution to cardiopulmonary failure. Peripheral arterial access is established by either vascular cutdown or percutaneously via a modified Seldinger technique. Deployed arterial cannulas range in sizes but are commonly $15 \mathrm{Fr}$ to $21 \mathrm{Fr}$ with the common femoral artery most frequently utilized given vessel size and ease of access. ${ }^{1}$ A prior study by Danial and colleagues ${ }^{2}$ found the percutaneous approach for obtaining vascular access for peripheral VA-ECMO was associated with fewer local infections and improved 30-day survival compared with surgical arterial cutdown. However, this study noted percutaneous access has been associated with higher rates of vascular complications at the time of decannulation. As a result, improvements in cannula removal techniques have been sought.

Due to the large arterial vascular sheath required for VAECMO, decannulation has traditionally been accomplished through surgical vascular repair with exposure and repair of the common femoral artery. Although commonly employed, this approach does involve morbidity from surgery and delayed mobility related to an open procedure. ${ }^{3}$ In an effort to reduce the rates of vascular complications at the time of VA-ECMO decannulation and to avoid the need for traditional surgical vascular repair, percutaneous techniques for closure of arterial cannulation sites utilizing suture-mediated closure systems have been employed by a number of centers. ${ }^{4-8}$ Likewise, suture-mediated closures systems have been utilized for safe and successful closure of large-bore venous and arterial access sites following a variety of elective vascular procedures including the elective use of VA-ECMO before lung transplantation. ${ }^{9-14}$ The Perclose ProGlide (Abbott Vascular, Abbott Park, Ill) is 1 such suture-mediated closure system. When applied to elective procedures, the preclose technique, where the suture device is deployed after initial access of the vessel and before significant upsizing of the access site, has been widely described and successfully used to achieve hemostasis in endovascular procedures with arterial sheaths as large as $24 \mathrm{Fr}^{15}$ Alternatively, a postclosure technique where the suture device is deployed after upsizing of the access site has also been described, but has the potential to be significantly more technically challenging and prone to excessive blood loss when attempting to deploy the suture device after large-bore arterial access such as provided with VA-ECMO. Although suture-mediate closure systems have been utilized in the postclosure repair of VA-ECMO arteriotomy sites, this technique is supported by limited data, is not yet widely employed, and the application of the preclosure technique of deployment has not been previously reported in patients placed on VA-ECMO for an urgent indication or with use of arterial ECMO cannulas larger than $15 \mathrm{Fr}$. In this study, we sought to compare procedural outcomes and complications of the preclosure technique delivered via the Perclose ProGlide suture-mediated closure system with surgical repair of the arterial access site in decannulation following percutaneous cannulation for VA-ECMO in the management of cardiogenic shock.

\section{METHODS}

\section{Study Population}

We performed a retrospective observational cohort study of consecutive patients aged 18 years or older who were managed with and then subsequently survived to decannulation from VA-ECMO for the treatment of cardiogenic shock between January 2015 and April 2020 at the Inova Fairfax Hospital in Fairfax, Va, a tertiary care referral center. The study was approved by the Institutional Review Board (No. 18-3317) at Inova Fairfax Hospital in January 2015 and the need for informed written consent for publication was waived. Patients managed with central ECMO or peripheral ECMO with arterial access site in a location other than the femoral artery were excluded. Patients cannulated for VA-ECMO, decannulated, and then subsequently recannulated were included in analysis as separate cases. Patients who underwent open surgical repair after preclosure device failure were analyzed as part of the preclosure group.

\section{General Management Protocol}

VA-ECMO was considered in cases of refractory cardiogenic shock. Percutaneous cannulation was performed in all instances using a modified Seldinger technique with a standard arterial cannula size ranging from $15 \mathrm{Fr}$ to $21 \mathrm{Fr}$. Ultrasound guidance was utilized universally during percutaneous access and efforts to minimize distal limb ischemia through the placement of a distal perfusion catheter were made in all circumstances where this was technically feasible. Site selection and the use of the preclosure technique at the time of arterial access were based on operator preference, comfort with the procedure, and availability of the preclosure devices. No clinical characteristics were considered strict contraindications to employment of this method. A diagram summarizing how patients were selected for the deployment of the preclosure device at our institution during the study period is provided in Figure 1. In instances where concern for significant atherosclerotic disease was high, fluoroscopic guidance was employed in addition to ultrasound guidance when clinically feasible. All VA-ECMO cannulations and decannulations were performed by an experienced cardiothoracic surgeon. During the study period at our institution, 6 total surgeons were in practice and all surgeons had significant experience and training in the evaluated techniques.

\section{Preclosure Technique}

Typically, in instances where the preclosure technique was utilized, femoral arterial access was obtained and a $6 \mathrm{Fr}$ dilator was inserted following guidewire insertion. Two Perclose ProGlide suture-mediated closure devices were then deployed using standard technique. After removing the second device and carefully securing the sutures to the skin with surgical strips; upsizing of the access point proceeded using standard techniques to accommodate the necessary arterial ECMO cannula.

During the study period, the preference was for decannulation from VAECMO to proceed in the operating room by the same surgeon who performed the initial cannulation (where clinically feasible). At the time of VA-ECMO decannulation a guidewire was advanced through the arterial 


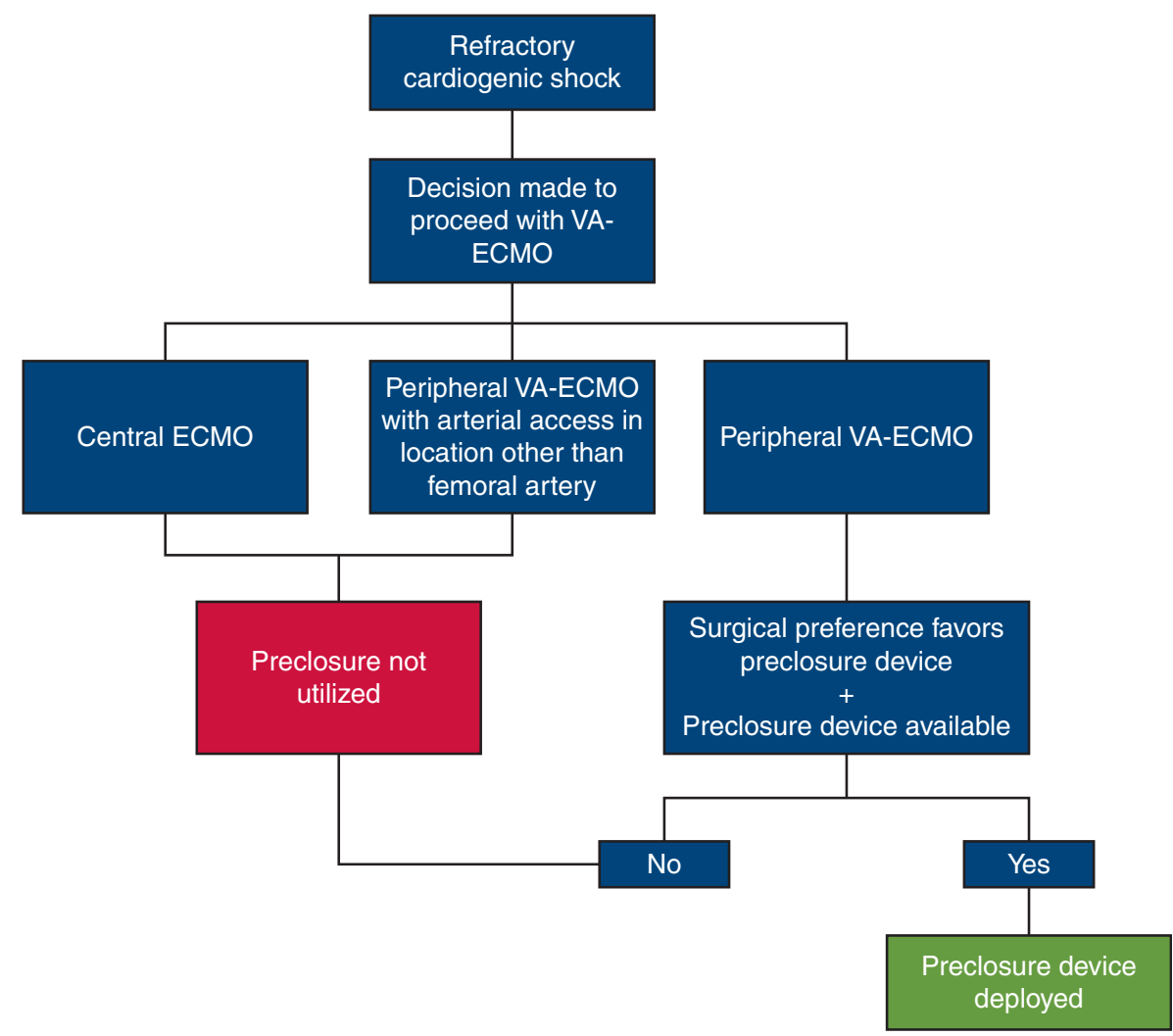

FIGURE 1. Summary of patient selection for the preclosure technique at our institution during the study period. VA-ECMO, Venoarterial extracorporeal membrane oxygenation.

cannula, the cannula was removed, and manual compression was applied by an assistant. The preloaded sutures were then secured in the standard fashion. With the guidewire in place, hemostasis was observed and if inadequate, a third device was deployed over the remaining guidewire. After confirming adequate hemostasis, the wire was removed and the Perclose ProGlide sutures were secured completely. If hemostasis was unacceptable after deployment of the third suture-mediated closure device, open repair was performed.

\section{Data Collection and Statistical Analysis}

Data were abstracted in a structured format by 2 of the authors (L.K.C. and M.D.). Data were collected from the electronic medical record (Epic; Epic Systems Corporation, Verona, Wis) from time of VA-ECMO initiation and until death or discharge from the intensive care unit. Baseline demographic information, clinical data, and relevant outcomes were collected. Percutaneous closure device failure was defined by inability to achieve hemostasis at the arteriotomy site necessitating adjuvant therapy extending beyond manual compression or arterial stenosis requiring endovascular or surgical intervention. The primary outcome examined was a composite of limb complications defined as the occurrence of limb ischemia within 24 hours following ECMO decannulation, limb infection (local inflammation, cellulitis, or purulence) at 48 hours, or groin site necrosis at 48 hours following ECMO decannulation. Secondary end points included bleeding events (bleeding noted at the cannulation site consistent with the Bleeding Academic Research Consortium Definition for Type 2 bleeding $^{16}$ ), pseudoaneurysm occurrence following decannulation, and distal part embolization within 48 hours of VA-ECMO weaning. The presence or absence of these events was based on the standardized surgical team documentation as per institutional VA-ECMO protocols. Intensive care unit (ICU) length of stay after decannulation from VA-ECMO was also examined.
Distribution of all continuous data was examined for normality using visual inspection and the Shapiro-Wilk test. Continuous data are presented as the median and interquartile range and compared using the Wilcoxon ranksum test. Characteristics of the groups are presented as counts with proportions and compared using Fisher exact test. Strength of associations between intervention and outcome are presented as odds ratios (ORs) calculated via exact logistic regression analysis. ICU length of stay demonstrated a positively skewed distribution. To minimize the effects of outliers and to account for this distribution, negative binomial regression was utilized to compare this outcome. Finally, to explore the effect of surgeon comfort and procedural urgency on outcomes, the analysis was repeated after the removal of patients with procedures performed by the surgeon with the highest number of preclosure procedures performed and those patients placed on ECMO during ongoing cardiopulmonary resuscitation, respectively. All relevant statistical tests were 2-tailed. All statistical analyses were performed using STATA version 14 (StataCorp LP, College Station, Tex).

\section{RESULTS}

Ninety-eight consecutive patients who survived to decannulation from peripheral VA-ECMO were identified during the study period. Two of these patients were decannulated and subsequently recannulated for VA-ECMO. One patient was excluded because arterial ECMO access was via the subclavian artery. Of those included $(\mathrm{N}=99), 2$ patients were managed with hybrid ECMO configurations and 31 patients $(32.3 \%)$ underwent additional cardiac unloading with placement of short-term cardiac assist devices (via 
the Impella [Abiomed, Danvers, Mass] heart pump per institutional preference). Ultimately, 51 patients had preclosure devices placed at the time of ECMO cannulation with successful hemostasis achieved following weaning and Perclose ProGlide deployment in $46(90.2 \%)$ of these patients. In the 5 instances of percutaneous closure device failure, open repair $(n=4)$ and balloon angioplasty $(n=1)$ were required. Alternatively, 48 patients did not have preclosure devices placed at the time of ECMO cannulation and subsequently underwent surgical removal at the time of decannualtion.

Baseline demographic and management characteristics of patients stratified by use of the preclosure device are displayed in Table 1 and were similar between the groups. The indications, management considerations, and surgeon who performed the cannulation are presented in Table 2. Of note, significant differences between individual surgeon's use of the preclosure technique was observed. Further, the preclosure device was utilized less frequently in patients cannulated while receiving ongoing cardiopulmonary resuscitation.

The clinical outcomes are summarized in Table 3. The primary outcome of limb complications occurred significantly more frequently in patients managed without the preclosure device compared with those where the device was utilized $(25.0 \%$ vs $5.9 \% ; P=.011)$. Increased frequency in limb complications was primarily driven by higher incidence of infection at the removal site; however, occurrence of limb ischemia and groin site necrosis were also numerically higher in the group where preclosure was not utilized.
Furthermore, significant bleeding events occurred more frequently in patients where the preclosure device was not applied $(22.9 \%$ vs $5.9 \% ; P=.020)$. ICU length of stay after decannulation was not significantly different between the groups and pseudoaneurysm and distal part embolization occurred infrequently in the cohort. Notably, severe complications such as systemic infection, loss of limb, or death were not observed.

The results of univariate exact logistic regression analysis relating preclosure use with the outcomes of interest are displayed in Table 4. Use of the preclosure device was associated with a nearly $80 \%$ reduction in limb complications (OR, 0.19; 95\% CI, 0.03-0.78) and bleeding events (OR, $0.21 ; 95 \%$ CI, 0.04-0.89). Use of the preclosure technique remained associated with reduced limb complications when patients cannulated during cardiopulmonary life support were removed from the analysis $(26.5 \%$ vs $6.4 \%$; $P=.023$ ) (Table 5). However, although a trend toward improved outcomes with the use of the preclosure technique was observed, no significant difference in outcomes between techniques was noted when data related to the surgeon who most frequently utilized the preclosure technique $(\mathrm{n}=24)$ were excluded (Table 6$)$.

\section{DISCUSSION}

Our study examined the procedural outcomes of the preclosure technique with the Perclose ProGlide suturemediated closure system versus arterial cutdown after percutaneous cannulation for VA-ECMO in the setting of advanced cardiogenic shock. In our cohort of consecutive

TABLE 1. Baseline characteristics of patients

\begin{tabular}{|c|c|c|c|c|}
\hline Characteristic & $\begin{array}{c}\text { All subjects } \\
\mathbf{n}=99\end{array}$ & $\frac{\text { Preclosure used }}{\mathrm{n}=51}$ & $\frac{\text { Surgical removal }}{n=48}$ & $P$ value \\
\hline \multicolumn{5}{|l|}{ Demographic data } \\
\hline Age (y) & $57(41,67)$ & $56(36,68)$ & $58(51,63)$ & .492 \\
\hline Female sex & $29(29.3)$ & $18(35.3)$ & $11(22.9)$ & .193 \\
\hline Body mass index & $28.0(23.7,32.7)$ & $27.1(22.8,34.2)$ & $28.4(24.3,31.8)$ & .504 \\
\hline \multicolumn{5}{|l|}{ Comorbid conditions } \\
\hline Hypertension & $48(48.5)$ & $22(43.1)$ & $26(54.2)$ & .317 \\
\hline Diabetes mellitus & $22(22.2)$ & $12(23.5)$ & $10(20.8)$ & .812 \\
\hline Coronary artery disease & $49(49.5)$ & $23(45.1)$ & $26(54.2)$ & .424 \\
\hline Peripheral arterial disease & $1(1.0)$ & 0 & $1(2.1)$ & .480 \\
\hline Chronic kidney disease & $12(12.1)$ & $4(7.8)$ & $8(16.7)$ & .225 \\
\hline End-stage renal disease & $7(7.1)$ & $2(3.9)$ & $5(10.4)$ & .259 \\
\hline \multicolumn{5}{|c|}{ Laboratory data at time of cannulation } \\
\hline INR & $1.5(1.2,2.1)$ & $1.4(1.1,1.9)$ & $1.7(1.3,2.3)$ & .066 \\
\hline Creatinine (mg/dL) & $1.4(1.0,2.1)$ & $1.4(0.9,2.0)$ & $1.6(1.0,2.4)$ & .223 \\
\hline Platelets $\left(\mathrm{L}^{-1}\right)$ & $183(118,246)$ & $183(129,271)$ & $177(105,231)$ & .417 \\
\hline \multicolumn{5}{|c|}{ Laboratory data at time of decannulation } \\
\hline INR & $1.2(1.1,1.3)$ & $1.1(1.1,1.3)$ & $1.2(1.1,1.3)$ & .325 \\
\hline Creatinine $(\mathrm{mg} / \mathrm{dL})$ & $1.1(0.8,1.9)$ & $1.1(0.8,1.7)$ & $1.25(0.8,2.15)$ & .128 \\
\hline Platelets $\left(\mathrm{L}^{-1}\right)$ & $107(71,147)$ & $107(80,147)$ & $107(62,148)$ & 690 \\
\hline
\end{tabular}

Values are presented as median (25th percentile, 75 th percentile) or $\mathrm{n}(\%)$. INR, International normalized ratio. 
TABLE 2. Indication and management characteristics of patients

\begin{tabular}{|c|c|c|c|c|}
\hline Variable & $\frac{\text { All subjects }}{n=99}$ & $\frac{\text { Preclosure used }}{\mathrm{n}=51}$ & $\frac{\text { Surgical removal }}{n=48}$ & $P$ value \\
\hline Indication for VA-ECMO & & & & .369 \\
\hline Left heart failure & $87(87.9)$ & $44(86.3)$ & $43(89.6)$ & \\
\hline Right heart failure & $6(6.1)$ & $5(9.8)$ & $1(2.1)$ & \\
\hline Acute pulmonary embolism & $6(6.1)$ & $3(5.9)$ & $3(6.3)$ & \\
\hline \multicolumn{5}{|l|}{ Management considerations } \\
\hline Cardiopulmonary resuscitation ongoing & $18(18.2)$ & $4(7.8)$ & $14(29.3)$ & .008 \\
\hline Arterial cannula size $(\mathrm{Fr})$ & $17(17,19)$ & $17(17,19)$ & $17.5(17,19)$ & .421 \\
\hline Ipsilateral arterial and venous catheters & $87(87.9)$ & $46(90.2)$ & $41(85.4)$ & .546 \\
\hline Percutaneous Impella* & $31(32.3)$ & $18(35.3)$ & $13(27.1)$ & .387 \\
\hline Time spent on ECMO (d) & $6(5,9)$ & $6(5,9)$ & $6(4.5,9)$ & .919 \\
\hline Surgeon performing cannulation $\dagger$ & & & & .047 \\
\hline $\mathrm{A}$ & 7 & 1 & 6 & \\
\hline $\mathrm{B}$ & 11 & 5 & 6 & \\
\hline $\mathrm{C}$ & 15 & 11 & 4 & \\
\hline $\mathrm{D}$ & 44 & 24 & 20 & \\
\hline E & 12 & 8 & 4 & \\
\hline $\mathrm{F}$ & 7 & 2 & 5 & \\
\hline OSH & 3 & 0 & 3 & \\
\hline
\end{tabular}

Values are presented as median (25th percentile, 75th percentile) or n (\%). VA-ECMO, Venoarterial extracorporeal membrane oxygenation; $O S H$, outside hospital. *Abiomed, Danvers, Mass. $\nmid$ Surgeon identified by coded letter, where $n$ reflects the number of procedures performed.

patients percutaneously placed on VA-ECMO who survived to decannulation, the use of the preclosure technique was associated with a significant decrease in limb complications and bleeding events with an estimated decreased likelihood of these complications of $81 \%$ and $79 \%$, respectively, compared with surgical removal (Figure 2). Furthermore, the percutaneous preclosure technique was frequently successful, with adequate hemostasis achieved in $90.2 \%$ of patients.

The preclosure technique has been demonstrated to be a simple, safe, and effective procedure for the repair of arteriotomy sites in elective procedures, including transcutaneous aortic valve replacement and endovascular aortic repair. Data from prior cohorts have documented the utility of this technique in the removal of arterial sheaths of sizes ranging from $5 \mathrm{Fr}$ to $24 \mathrm{Fr} .{ }^{9-15}$ However, previous data do not exist regarding the application of the preclosure technique to decannulation from VA-ECMO in the management of cardiopulmonary failure requiring urgent mechanical support. Innovative techniques and protocols aimed at utilizing suture-mediated closure systems in a postclosure fashion have been proposed and applied to VA-ECMO decannulation. However, these techniques have not yet demonstrated improvements in clinical outcomes compared with surgical removal in this setting. ${ }^{4-8}$ We suspect postclosure compared with preclosure may result in a more technically challenging VA-ECMO decannulation procedure and be prone to excessive blood loss when attempting to deploy the suture device after large-bore arterial access such as provided with VA-ECMO. It has been suggested that a possible

TABLE 3. Primary and secondary outcomes of patients by use of preclosure device

\begin{tabular}{|c|c|c|c|c|}
\hline Outcome & All subjects & Preclosure used & Surgical removal & $P$ value \\
\hline \multicolumn{5}{|l|}{ Primary outcome } \\
\hline Limb complications & $15(15.2)$ & $3(5.9)$ & $12(25.0)$ & .011 \\
\hline \multicolumn{5}{|l|}{ Components of primary outcome } \\
\hline Limb ischemia & $7(7.1)$ & $2(3.9)$ & $5(10.4)$ & .259 \\
\hline Infection at removal site & $8(8.1)$ & $1(2.0)$ & $7(14.6)$ & .028 \\
\hline Removal site necrosis & $2(2.0)$ & 0 & $2(4.2)$ & .233 \\
\hline \multicolumn{5}{|l|}{ Secondary outcomes } \\
\hline Bleeding event & $14(14.1)$ & $3(5.9)$ & $11(22.9)$ & .020 \\
\hline Pseudoaneurysm & 0 & 0 & 0 & - \\
\hline Distal part embolization & $1(1.0)$ & 0 & $1(2.1)$ & .485 \\
\hline ICU length of stay after decannulation (d) & $9(5,17)$ & $8(5,17)$ & $9(5,20)$ & .725 \\
\hline
\end{tabular}

Values are presented as median (25th percentile, 75 th percentile) or $\mathrm{n}(\%)$. ICU, Intensive care unit. 
TABLE 4. Analysis of outcomes of patients stratified by use of preclosure device (surgical removal as reference) with adjustment for confounders

\begin{tabular}{lcc}
\hline \multicolumn{1}{c}{ Outcome } & $\begin{array}{c}\text { Odds ratio } \\
(\mathbf{9 5} \% \text { confidence interval)* }\end{array}$ & $\boldsymbol{P}$ value \\
\hline $\begin{array}{l}\text { Primary outcome } \\
\text { Limb complications }\end{array}$ & $0.19(0.03-0.78)$ & .016 \\
$\begin{array}{l}\text { Secondary outcomes } \\
\text { Bleeding event }\end{array}$ & $0.21(0.04-0.89)$ & .030 \\
ICU length of stay & $0.84(0.58-1.20) \dagger$ & .329 \\
\hline
\end{tabular}

ICU, Intensive care unit. *Statistical comparison performed using exact logistic regression analysis. $\dagger$ Incident relative ratio by negative binomial regression analysis.

advantage of the postclosure technique compared with preclosure in VA-ECMO is that, given the uncertain duration of extracorporeal therapy, suture-mediated system stitches may remain in place and be subject to infection for prolonged periods of time. However, site infection after cannula removal was uncommon $(2.0 \%)$ in the preclosure group of our cohort and this rate was comparable to those demonstrated in postclosure following decannulation of VA-ECMO ${ }^{4,6,7}$ Further, in a previous study of the use of the preclosure technique in elective peripheral VA-ECMO before lung transplantation, similarly low rates of site infections were noted. ${ }^{14}$

Historically, it has been typical to perform surgical vascular repair with exposure and repair of the common femoral artery at the time of decannulation from VAECMO. This open surgical procedure was associated with the risks of an open operation, related decreased patient mobility following its completion, and potential for seroma formation. Given our center's experience, it has now become standard at our institution to utilize the preclosure technique via a suture-mediated closure device in the methods described in this study for patients considered for VA-ECMO. This description of our experience documents preclosure use experience over a 5-year interval. Notably, provider experience and procedural mastery has increased over time and although 5 suture-mediated closure device failures were noted in our cohort, all failures occurred early in our institutional experience and we anticipate an even lower failure rate over time. Whereas foresight, planning, and operator skill and experience is required at the time of VA-ECMO initiation to deploy the Perclose ProGlide suture-mediated closure system before upsizing of the arterial access point, the minimal extra time spent deploying this system has not been found, in our experience, to unnecessary prolong time to initiation of extracorporeal therapy in critically ill patients. Our typical technique involves the deployment of 2 suturemediated closure systems at the time of cannulation. This double technique allows for redundancy in the event of system failure but does modestly increase procedural duration. Of note, we have found the deployment of a third Perclose ProGlide device at the time of decannulation is very rarely necessary to achieve adequate hemostasis (Video 1).

This analysis has a few limitations. First, this was a retrospective observational study. Although the 2 compared groups were well balanced based on most measured confounders, all confounders could not be accounted for given the small sample size and retrospective analysis of a procedure performed under urgent conditions in a critically ill population. Further, some secondary vascular complications such as pseudoaneurysm and distal part embolization occurred infrequently in both groups. Data from larger cohorts are required to identify differences, if any, in outcomes for these complications. Alternatively, bleeding events occurred frequently in the surgical decannulation group $(22.9 \%)$. This high rate of bleeding may have been related to ongoing systemic anticoagulation at the time of the open surgical procedure as required for ongoing Impella device support. Not all observed bleeding events may have been clinically relevant. Patient specific factors such as significant peripheral artery disease or anatomic considerations related to the access site could not be fully examined before arterial access or accounted for in data analysis given the urgent nature of the procedure. Significant calcific disease in the arterial access point has been noted to be associated with worse outcomes related to preclosure deployment in other cohorts. ${ }^{17}$ However, the effects of this could not be examined given the impracticality of obtaining preprocedural imaging, but may have had limited effect in this population given that the strict eligibility criteria for VA-ECMO initiation may have naturally excluded patients with significant preexisting vascular disease. Further, whereas the rate of late vessel stenosis when suture-mediated closure devices are utilized for other indications has been reported to be low, long-term vascular outcomes related to preclosure device deployment for ECMO remains unknown. ${ }^{18}$ Additionally, patients that died before ECMO decannulation were

TABLE 5. Subgroup analysis of outcomes of patients by use of preclosure device with patients that were cannulated during ongoing cardiopulmonary resuscitation removed

\begin{tabular}{lcccc}
\hline \multicolumn{1}{c}{ Outcome } & All subjects & Preclosure used & Surgical removal & $\boldsymbol{P}$ value \\
\hline Limb complications & $12(14.8)$ & $3(6.4)$ & $9(26.5)$ & .023 \\
Bleeding event & $10(12.3)$ & $3(6.4)$ & $7(20.6)$ & .086 \\
ICU length of stay after decannulation (d) & $10(5,20)$ & $10(5,20)$ & $9.5(5,21)$ & .618 \\
\hline
\end{tabular}

Values are presented as median (25th percentile, 75 th percentile) or $\mathrm{n}(\%)$. ICU, Intensive care unit 
TABLE 6. Subgroup analysis of outcomes of patients by use of preclosure device with patients that were cannulated by the most experienced surgeon removed

\begin{tabular}{lcccc}
\hline \multicolumn{1}{c}{ Outcome } & All subjects & Preclosure used & Surgical removal & $\boldsymbol{P}$ value \\
\hline Limb complications & $4(7.3)$ & $0(0)$ & $4(14.3)$ & .111 \\
Bleeding event & $5(9.1)$ & $0(0)$ & $5(17.9)$ & .051 \\
ICU length of stay after decannulation (d) & $10(5,21)$ & $11(5,20)$ & $9(5,23.5)$ & .860 \\
\hline
\end{tabular}

Values are presented as median (25th percentile, 75 th percentile) or $\mathrm{n}(\%)$. ICU, Intensive care unit.

not included in data collection given our goal to compare outcomes related to 2 different decannulation procedures. Therefore, we are unable to assess the association of the perclosure device and patient outcomes while receiving ECMO support. Conceivably, the additional time required for deployment of the preclosure device before ECMO support could have a detrimental effect on outcomes related to the management of cardiogenic shock. This effect, if present, may be most significant in patients cannulated for ECMO support on the most urgent basis. Finally, although use of the preclosure technique has expanded over time at our institution, ultimately, procedural selection has been based on surgeon comfort and preference. Further, decision to utilize the preclosure technique may have been influenced by perceived clinical urgency of VA-ECMO initiation. Conceivably, given time constraints, avoidance of the use of the preclosure device and increased vascular injury at the time of arterial cannulation may have occurred in patients where arterial access was achieved on the most urgent basis. Both surgical preference and patient selection are possible sources of bias in this analysis and are evidenced by the difference in techniques applied by individual surgeons and by the diminished utilization of the preclosure technique during cannulation of patients receiving cardiopulmonary resuscitation. We attempted to account for these sources of bias through a subgroup analysis with the removal of patients cannulated during ongoing cardiopulmonary resuscitation and with the removal of patients cannulated by the most experienced surgeon. Although a trend toward improved outcomes persisted, these results should be cautiously interpreted given the limited remaining sample size in these analyses. Despite these considerations, we note that data related to the use of the preclosure technique in relation to VA-ECMO is lacking and thus, believe the outcomes noted in this cohort are of clinical importance.

\section{CONCLUSIONS}

In this cohort of consecutive patients treated with VAECMO for cardiogenic shock, a total percutaneous approach to cannulation and decannulation utilizing a

\section{Preclosure technique versus arterial cutdown after percutaneous cannulation for venoarterial extracorporeal membrane oxygenation}

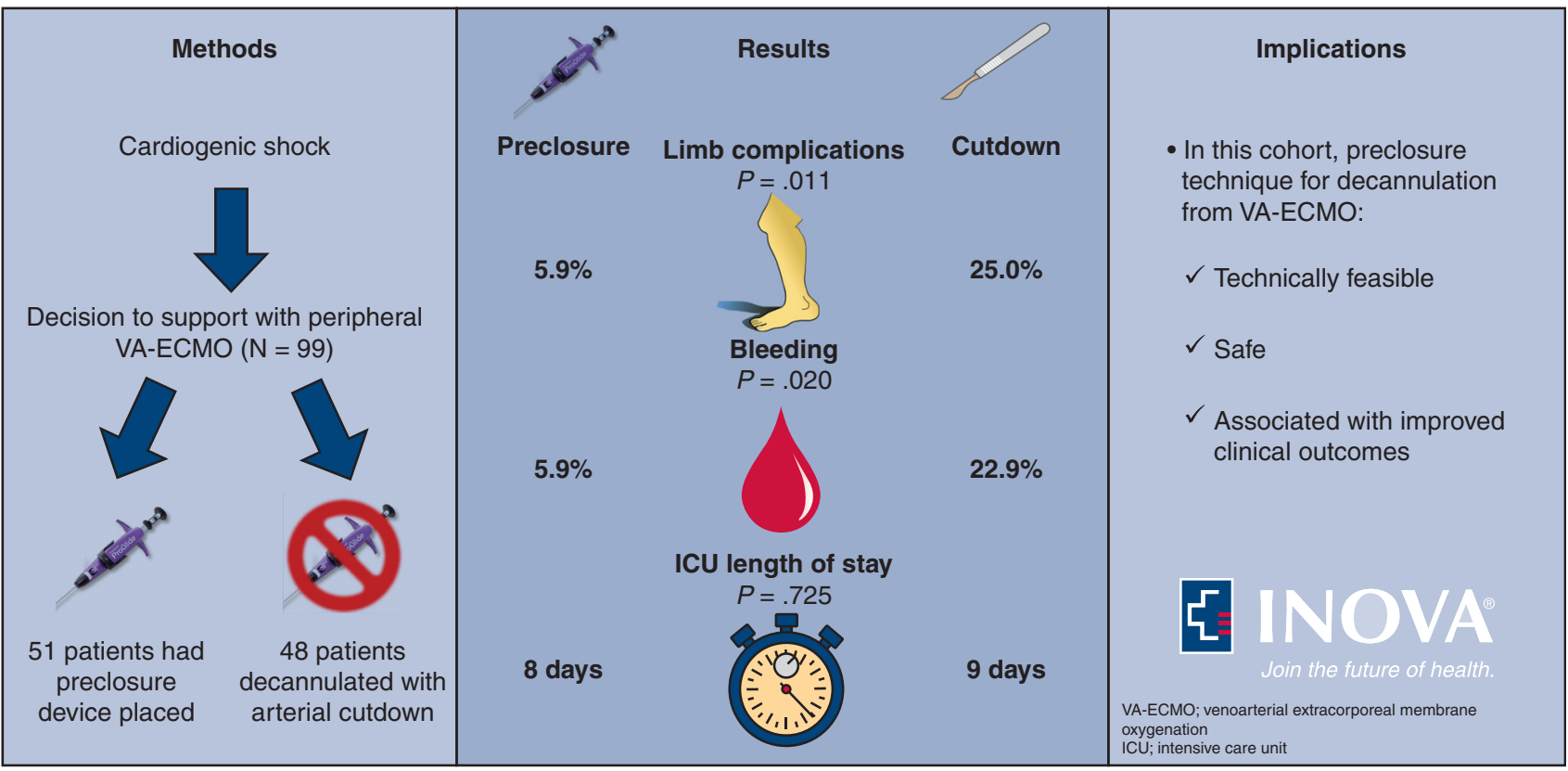

FIGURE 2. A totally percutaneous approach of cannulation and decannulation from venoarterial extracorporeal membrane oxygenation (VA-ECMO) utilizing a preclosure technique was technically feasible, safe, and associated with improved vascular outcomes. ICU, Intensive care unit. 


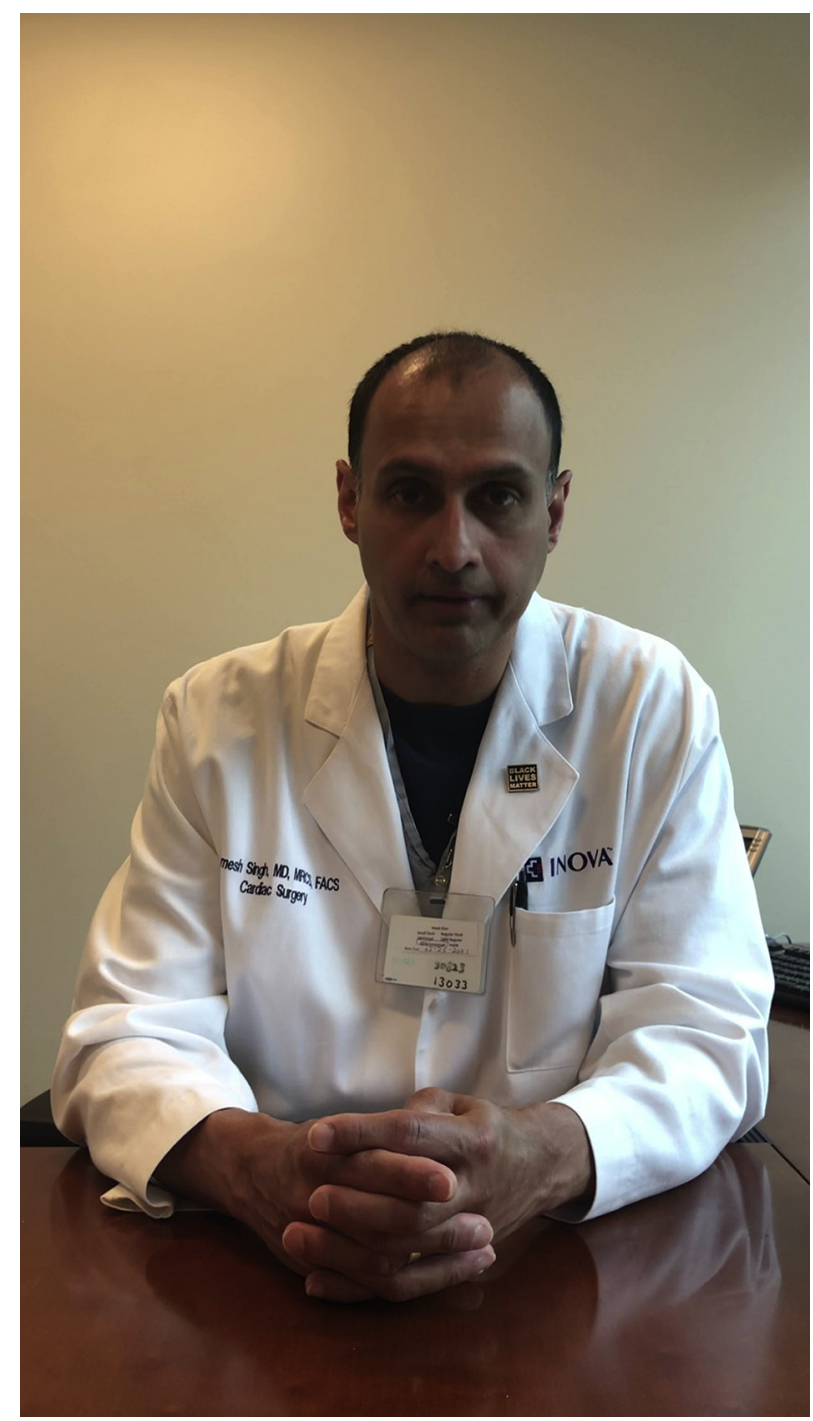

VIDEO 1. Dr Ramesh Singh, Surgical Director of Mechanical Circulatory Support at the Inova Heart and Vascular Institute, discussing the results and clinical implications of a totally percutaneous approach of cannulation and decannulation from VA-ECMO utilizing a preclosure technique. Video available at: https://www.jtcvs.org/article/S2666-2507(21)00589-7/ fulltext.

preclosure technique with the Perclose ProGlide suturemediated closure system was technically feasible, safe, and associated with significant lower likelihood of limb complications and bleeding. Prospective evaluation of these findings will be helpful to define the optimal procedural protocol for implementation of this surgical technique in this clinical setting.

\section{Conflict of Interest Statement}

Dr Chandel is an employee of Walter Reed National Military Medical Center, Bethesda, MD. The views expressed in this article are those of the author and do not reflect the official policy of the Department of Army/Navy/Air Force, Department of Defense (DOD), or the US Government. The identification of specific products is considered an integral part of the scientific endeavor and does not constitute endorsement or implied endorsement on the part of the author, DoD, or any component agency. All other authors reported no conflicts of interest.

The Journal policy requires editors and reviewers to disclose conflicts of interest and to decline handling or reviewing manuscripts for which they may have a conflict of interest. The editors and reviewers of this article have no conflicts of interest.

\section{References}

1. Rao P, Khalpey Z, Smith R, Burkhoff D, Kociol RD. Venoarterial extracorporeal membrane oxygenation for cardiogenic shock and cardiac arrest. Circ Heart Fail. 2018:11:e004905.

2. Danial P, Hajage D, Nguyen LS, Mastroianni C, Demondion P, Schmidt M, et al. Percutaneous versus surgical femoro-femoral veno-arterial ECMO: a propensity score matched study. Intensive Care Med. 2018;44:2153-61.

3. Aziz F, Brehm CE, El-Banyosy A, Han DC, Atnip RG, Reed AB. Arterial complications in patients undergoing extracorporeal membrane oxygenation via femoral cannulation. Ann Vasc Surg. 2014;28:178-83.

4. Au SY, Chan KS, Fong KM, Leung PR, Ng WG, So SO, et al. One-year experience of bedside percutaneous VA-ECMO decannulation in a high-ECMO-volume center in Hong Kong. Perfusion. 2020; 267659120971998.

5. Lusebrink E, Stremmel C, Stark K, Lüsebrink E, Stremmel C, Stark K, et al Percutaneous decannulation instead of surgical removal for weaning after venoarterial extracorporeal membrane oxygenation-a crossed Perclose ProGlide closure device technique using a hemostasis valve Y connector. Crit Care Explor. 2019;1:e018.

6. Hwang JW, Yang JH, Sung K, Song YB, Hahn JY, Choi JH, et al. Percutaneous removal using Perclose ProGlide closure devices versus surgical removal for weaning after percutaneous cannulation for venoarterial extracorporeal membrane oxygenation. J Vasc Surg. 2016;63:998-1003.e1.

7. Majunke N, Mangner N, Linke A, Boudriot E, Erbs S, Tietz F, et al. Comparison of percutaneous closure versus surgical femoral cutdown for decannulation of large-sized arterial and venous access sites in adults after successful weaning of veno-arterial extracorporeal membrane oxygenation. J Invasive Cardiol. 2016;28:415-9.

8. Shah A, Ghoreishi M, Taylor BS, Toursavadkohi S, Kaczorowski DJ, University of Maryland Medical Center Extracorporeal Membrane Oxygenation Group. Complete percutaneous decannulation from femoral venoarterial extracorporeal membrane oxygenation. J Thorac Cardiovasc Surg Tech. 2020;6:75-81.

9. Saadi EK, Saadi M, Saadi R, Tagliari AP, Mastella B. Totally percutaneous access using perclose proglide for endovascular treatment of aortic diseases. Braz J Cardiovasc Surg. 2017;32:43-8.

10. Lozano I, Avanzas P, Moris C. On-site immediate removal of intraortic balloon pump after high-risk percutaneous intervention with a 6Fr closure suture device with the "preclosure" technique. Catheter Cardiovasc Interv. 2007;70: 538-40.

11. Van Mieghem NM, Latib A, van der Heyden J, van Gils L, Daemen J, Sorzano T, et al. Percutaneous plug-based arteriotomy closure device for large-bore access: a multicenter prospective study. JACC Cardiovasc Interv. 2017;10:613-9.

12. Griese DP, Reents W, Diegeler A, Kerber S, Babin-Ebell J. Simple, effective and safe vascular access site closure with the double-ProGlide preclose technique in 162 patients receiving transfemoral transcatheter aortic valve implantation. Catheter Cardiovasc Interv. 2013;82:E734-41.

13. Hamid T, Rajagopal R, Pius C, Clarke B, Mahadevan VS. Preclosure of largesized venous access sites in adults undergoing transcatheter structural interventions. Catheter Cardiovasc Interv. 2013;81:586-90.

14. Pellenc Q, Girault A, Roussel A, Aguir S, Cerceau P, Longrois D, et al. Preclosing of the femoral artery allows total percutaneous venoarterial extracorporeal membrane oxygenation and prevents groin wound infection after lung transplantation. Eur J Cardiothorac Surg. 2020;58:371-8. 
15. Lee WA, Brown MP, Nelson PR, Huber TS, Seeger JM. Midterm outcomes of femoral arteries after percutaneous endovascular aortic repair using the Preclose technique. J Vasc Surg. 2008;47:919-23.

16. Mehran R, Rao SV, Bhatt DL, Gibson CM, Caixeta A, Eikelboom J, et al. Standardized bleeding definitions for cardiovascular clinical trials: a consensus report from the Bleeding Academic Research Consortium. Circulation. 2011;123:2736-47.

17. Lee CH, Ko YG, Park Y, Shim CY, Hong GR, Lee SH, et al. Risk factors for closure failure following percutaneous transfemoral transcatheter aortic valve implantation. Ann Vasc Surg. 2020;66:406-14.
18. Hu G, Chen B, Fu W, Xu X, Guo D, Jiang J, et al. Predictors and treatments of proglide-related complications in percutaneous endovascular aortic repair. PLoS One. 2015;10:e0123739.

Key Words: extracorporeal membrane oxygenation, percutaneous arterial closure, arteriotomy repair, arterial complications, cardiogenic shock 